\title{
Towards new vistas in preamplifier design for MRI
}

Johansen, Daniel Højrup; Sanchez, Juan Diego; Zhurbenko, Vitaliy; Ardenkjær-Larsen, Jan Henrik

Published in:

Proceedings of the 47th European Microwave Conference

Publication date:

2017

Document Version

Peer reviewed version

Link back to DTU Orbit

Citation (APA):

Johansen, D. H., Sanchez, J. D., Zhurbenko, V., \& Ardenkjær-Larsen, J. H. (2017). Towards new vistas in preamplifier design for MRI. In Proceedings of the 47th European Microwave Conference (pp. 1159-1162). IEEE.

\section{General rights}

Copyright and moral rights for the publications made accessible in the public portal are retained by the authors and/or other copyright owners and it is a condition of accessing publications that users recognise and abide by the legal requirements associated with these rights.

- Users may download and print one copy of any publication from the public portal for the purpose of private study or research.

- You may not further distribute the material or use it for any profit-making activity or commercial gain

- You may freely distribute the URL identifying the publication in the public portal

If you believe that this document breaches copyright please contact us providing details, and we will remove access to the work immediately and investigate your claim. 


\title{
Towards New Vistas in Preamplifier Design for MRI
}

\author{
Daniel H. Johansen ${ }^{1}$, Juan D. Sanchez-Heredia ${ }^{2}$, Vitaliy Zhurbenko ${ }^{3}$, and Jan H. Ardenkjær-Larsen ${ }^{4}$ \\ Technical University of Denmark, Department of Electrical Engineering \\ Ørsteds Plads, Building 349, 2800 Kgs. Lyngby, Denmark \\ Emails: $\left\{{ }^{1}\right.$ dhjo, ${ }^{2}$ jdsanch, ${ }^{3}$ vz, ${ }^{4}$ jhar $\} @$ elektro.dtu.dk
}

\begin{abstract}
High signal to noise ratio (SNR) in magnetic resonance imaging is vital for ensuring accurate diagnosis and treatment. Arrays of surface coils for receive only purposes is a well established way to increase SNR. However, due to crosstalk between the array elements, the SNR can be severely degraded. For that reason, arrays often do not exploit their full potential. By using a series decoupling network with non-conventional matching and preamplifier impedances the decoupling between elements can be increased significantly. In the presented design example, almost $6 \mathrm{~dB}$ additional decoupling can be achieved with no impairment of preamplifier noise figure. The decoupling changes as a function of both coil and preamplifier performance. Thus, the fundamental trade-off between noise and decoupling is discussed. This work embarks on the path towards new vistas in design of preamplifiers for surface coil arrays for magnetic resonance imaging.
\end{abstract}

Index Terms-Decoupling, matching networks, noise matching, preamplifiers, surface coils.

\section{INTRODUCTION}

Magnetic Resonance Imaging (MRI) is a non-invasive, nonionizing method that produces high resolution images of anatomy and physiology, while also being able to investigate metabolism by use of spectroscopy. This makes it a powerful tool for diagnostic of diseases like cancer and injuries such as broken limbs. Evaluating images is inherently a subjective task where the signal to noise ratio (SNR) is one of the prevalent measures of quality [1]. The SNR of the nuclear magnetic resonance experiment is limited. A low SNR impairs both imaging and acquisition speed, in turn increasing cost [1]. This work investigates the fundamental trade-off between noise and decoupling when an array of surface coils is utilized.

In its most basic form MRI polarizes nuclear spins (typically protons, ${ }^{1} \mathrm{H}$ ) within the patient in a strong static magnetic field called the main field. Now, the proton spins are tilted by applying an RF pulse orthogonally to the main field, at the Larmor frequency (precession frequency). When the RF pulse is switched off the proton spins return to equilibrium (relaxation). The precession and relaxation is recorded typically by Faraday induction. [2]

Several approaches can be used to increase SNR, including increasing the main field strength, longer scanning time by averaging and/or increasing the Q-factor of the receiving coil [3]. This work is focused on an approach using arrays of surface coils.

The key challenge in arrays is the parasitic coupling between elements. The coupling between elements cause a two fold impairment. 1) Degradation of the Q-factor of the individual coils [4]. Unless meticulously designed, more noise arises from the coupling between elements in an array than the loading of a volume coil by a patient. Hence, using surface coil arrays becomes void. 2) In the realm of parallel imaging coupling causes information correlation and thus limits the usefulness of parallel imaging [5]. Information correlation is meant in the sense that if two coils were perfectly coupled they would show the exact same image at all times.

This work focuses on preamplifier decoupling by extending the seminal work by Roemer et al. [4]. Specifically, how to choose the matching impedance in order to maintain proper noise matching of the preamplifier while maximizing the decoupling. Roemer et al. describes the series decoupling network where matching and minimizing the current in the coil is achieved simultaneously. Roemer et al., however, only demonstrated the decoupling in the $50 \Omega$ case. In this work, the fundamental trade-off between noise and decoupling is described when preamplifier decoupling is utilized. Further, it is shown that transforming the coil impedance to a complex impedance, rather than $50 \Omega$, leads to an increased decoupling while conserving the noise figure of the preamplifier.

The article is structured as follows. The first section reviews methods for coil decoupling. The second section derives the formulas describing the decoupling circuit. The third section describes the results and some practicalities in building and tuning the decoupling circuit. Lastly, conclusions are drawn.

\section{Coupled Surface CoIls}

Fig. 1 shows a system of two coils each attached with a matching circuit and preamplifier. The impedance of the coil is described by $Z_{\mathrm{C}}$. The impedance seen by the coil is $Z_{\mathrm{L}}$, the matching impedance seen by the preamplifier is $Z_{\mathrm{M}}$ and the preamplifier impedance is $Z_{\mathrm{P}}$. The coupling between the two coils is described by $j \omega M$ where $M$ is the mutual inductance and $\omega$ is the angular frequency. The current in the first coil $i_{1}$ when $i_{0} \neq 0$ is

$$
i_{1}=\frac{j \omega M}{Z_{\mathrm{C}}+Z_{\mathrm{L}}} i_{0}=\frac{j \omega k \sqrt{L_{0} L_{1}}}{Z_{\mathrm{C}}+Z_{\mathrm{L}}} i_{0},
$$

where $0 \leq k \leq 1$ is the coupling factor and $L$ is the inductance of the coils. Decoupling is, in most cases, achieved by using one or a combination of the following techniques.

1) Critical overlapping: When two surface coils are separated by a given distance a complete decoupling occurs [4]. This corresponds to $k=0$. This is the most used method for nearest neighbour decoupling. However, it does not work for next nearest neighbours. Another 


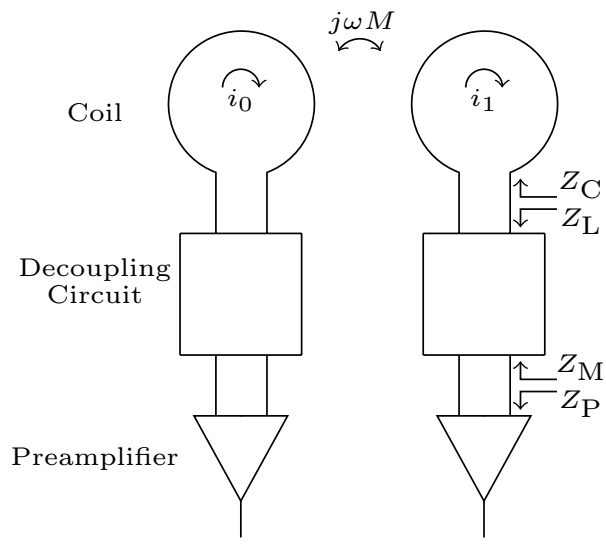

Figure 1. System of two coupled surface coils.

aspect of critical overlapping is that in parallel imaging the overlap causes two coils to be correlated. This impairs the reconstruction in parallel imaging [5].

2) Y-method: Choosing $Z_{\mathrm{L}}$ such that the mutual inductance is cancelled out, full decoupling is achieved. Most often a capacitor is simply added between elements but more complicated methods can be employed [6]-[8]. The problem with this method is that the complexity, for a larger number of elements, grows quickly, and thus has not been used for much more than research purposes in MRI.

3) Preamplifier decoupling: By increasing $Z_{\mathrm{L}}$ the current $i_{1}$ is lowered, and hence decoupling is again accomplished. This is very simple, can be easily tuned in and works for all elements in an array.

The most used methods are critical overlapping and preamplifier decoupling [4], [9].

\section{DeCOUPLing CiRCuit}

The general solution for the series decoupling circuit in Fig. 2 is presented when the matching and preamplifier impedances are both complex. Also, the matching impedance is discussed in terms of the noise parameters of the preamplifier.

\section{A. Series Decoupling Network}

The series decoupling network described by Roemer et al. is seen in Fig. 2. Roemer et al. derives the impedance for a real matching and preamplifier impedance leading to a simpler interpretation of the network than is merited for complex impedances. The series connection of the coil and $C_{1}$ is

$$
Z_{1}=R_{1}+j\left(X_{L_{1}}-X_{C_{1}}\right)
$$

The admittance $Y_{2}$ is

$$
Y_{2}=G_{2}+j B_{2}=\frac{1}{Z_{2}}=\frac{1}{Z_{1}}+j B_{C_{2}},
$$

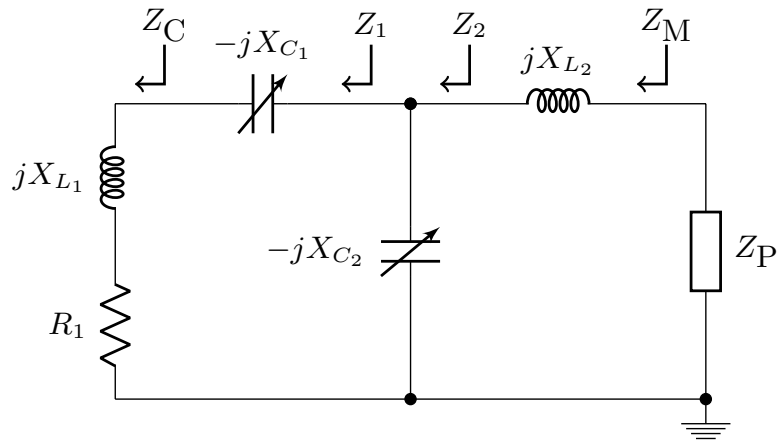

Figure 2. Circuit diagram of the series decoupling network as proposed by Roemer et al [4].

where $G_{2}$ and $B_{2}$ is the conductance and susceptance of $Y_{2}$ respectively and $B_{C_{2}}$ is the susceptance of $X_{C_{2}}=\frac{1}{B_{C_{2}}}$. Separating in real and imaginary components yield,

$$
\begin{aligned}
G_{2} & =\frac{R_{1}}{R_{1}^{2}+\left(X_{L_{1}}-X_{C_{1}}\right)^{2}}, \\
B_{2} & =\frac{X_{L_{1}}-X_{C_{1}}}{R_{1}^{2}+\left(X_{L_{1}}-X_{C_{1}}\right)^{2}}+B_{C_{2}} .
\end{aligned}
$$

The matching impedance is determined by

$$
Z_{\mathrm{M}}=R_{\mathrm{M}}+j X_{\mathrm{M}}=Z_{2}+j X_{L_{2}} .
$$

Separating into real and imaginary components yield

$$
\begin{aligned}
R_{\mathrm{M}} & =\frac{G_{2}}{G_{2}^{2}+j B_{2}^{2}}, \\
X_{\mathrm{M}} & =X_{L_{2}}-\frac{B_{2}}{G_{2}^{2}+j B_{2}^{2}} .
\end{aligned}
$$

By ensuring resonance of $C_{2}$ and $L_{2}$, ideally $Z_{\mathrm{L}}=\infty$. The required condition is

$$
X_{L_{2}}+X_{\mathrm{P}}=X_{C_{2}} \text {. }
$$

Thus Eqs. 7, 8 and 9 form a system of three equations with three unknowns and is solved by combining Eqs. 5, 4, 7, 8 and 9. The solution is

$$
\begin{aligned}
A & =\frac{\sqrt{R_{1} R_{\mathrm{M}}\left(R_{\mathrm{M}}^{2}+X_{\mathrm{M}}^{2}+2 X_{\mathrm{M}} X_{\mathrm{P}}+X_{\mathrm{P}}^{2}\right)}}{R_{\mathrm{M}}} \\
X_{C_{1}} & =\frac{R_{1}\left(X_{\mathrm{M}}+X_{\mathrm{P}}\right)}{R_{\mathrm{M}}}+X_{L_{1}} \mp A \\
X_{L_{2}} & =-X_{\mathrm{P}} \pm A,
\end{aligned}
$$

Positive results imply that $C_{1}, C_{2}$ are capacitors and $L_{2}$ is an inductor. If negative, the capacitors become inductors and vice versa. Interpreting the solution in the Smith Chart Fig. 3 clarifies. Given an inductance with some resistance the first point is (a). Adding a large series capacitor $C_{1}$ the reactance is moved only slightly to (b). By a parallel capacitor $C_{2}$ the impedance is transformed in a constant conductance circle into (c). From here, $C_{2}$ is resonated with $L_{2}$ transforming into $50 \Omega$ at (d). Another solution is to have a small $C_{1}$ transforming the 


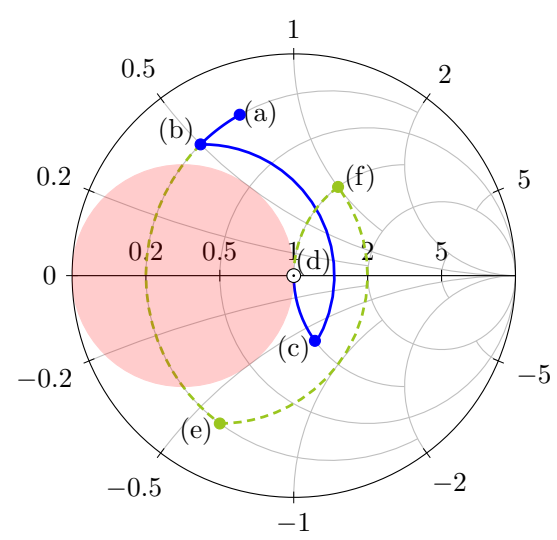

Figure 3. Smith Chart illustrating the two solutions of the series decoupling network.

impedance into the capacitive area of the Smith Chart, point (e). From here a parallel inductor is needed to transform to point (f). Finally, a series capacitor resonates the inductor and transforms to (d). Hence two solutions are indeed possible.

\section{B. Matching and Preamplifier impedances}

In Table I the properties of a $300 \mathrm{MHz}$ preamplifier are listed. The preamplifier is based on [10] but matched for 300 $\mathrm{MHz}$ instead of $32 \mathrm{MHz}$. This operating frequency corresponds to a main field strength of $7 \mathrm{~T}$. The noise figure as a function of the matching admittance is

$$
F=F_{\min }+\frac{R_{\mathrm{n}}}{G_{\mathrm{M}}}\left|Y_{\mathrm{M}}-Y_{\mathrm{n}}\right|^{2}
$$

where $F$ is the noise figure at the given matching admittance $Y_{\mathrm{M}}=G_{\mathrm{M}}+j B_{\mathrm{M}}=\frac{1}{Z_{\mathrm{M}}}, F_{\min }$ is the minimal noise figure at the optimal noise admittance $Y_{\mathrm{n}}$, and $R_{\mathrm{n}}$ is the noise resistance. Choosing $Y_{\mathrm{M}} \neq Y_{\mathrm{n}}$ yields a system of noise figure circles which can be visualized using a Smith Chart. Fig. 4 shows a circle in which the $50 \Omega$ impedance is located (on this circle all impedances yield the same noise figure, however, they do not yield the same decoupling). The optimal noise match is shown as a cross. The current $i_{1}$ is plotted relative to $i_{1}$ at $50 \Omega$ in Fig. 5 as a function of the angle of the noise figure circle. Each angle corresponds to a complex impedance. At $50 \Omega$ matching impedance the current is normalized to $0 \mathrm{~dB}$. The minimum current occurs at a matching impedance of $55+j 153 \Omega$. Here a $5.6 \mathrm{~dB}$ additional decoupling is achievable.

The gain of the preamplifier has not been discussed in much detail. The required gain for a preamplifier is defined by a

Table I

PREAMPLIFIER PROPERTIES.

\begin{tabular}{c|c}
$F_{\min }$ & 1.076 \\
\hline$R_{\mathrm{n}}$ & $11.60 \Omega$ \\
\hline$Y_{\mathrm{n}}$ & $0.00583-\mathrm{j} 0.00988 \Omega$ \\
\hline$Z_{\mathrm{P}}$ & $5.42-\mathrm{j} 57.11 \Omega$
\end{tabular}

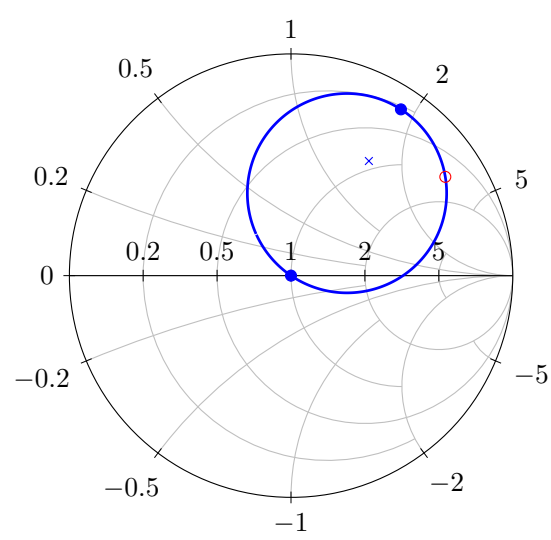

Figure 4. Noise figure circle passing through $50 \Omega$. Cross indicates optimal noise match, red open circle is the optimal decoupling. Anything between the closed circles on the optimal decoupling side indicate an increased decoupling.

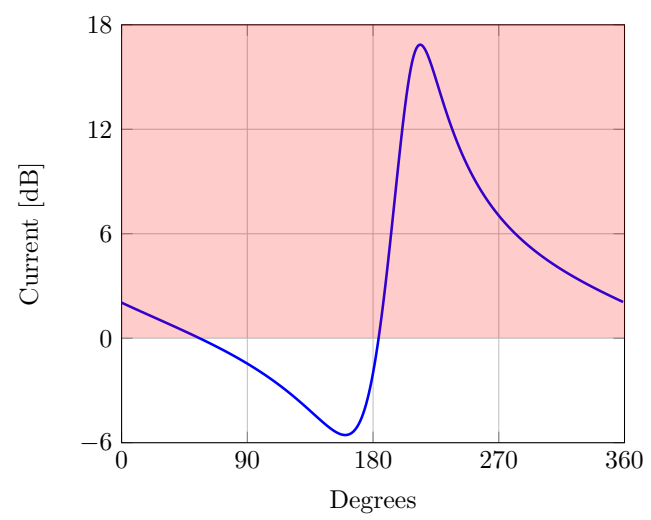

Figure 5. Current $i_{1}$ is relative to $i_{1}$ at $50 \Omega$ as a function of the angle of the noise figure circles corresponding to a unique impedance for each angle.

particular MRI system configuration and corresponding noise budget. Whatever the gain requirement is, the gain circles can be plotted in the same Smith Chart as the decoupling and noise figure circles. Their unity then defines the trade-off that can be made within the wanted requirements. Further work could look into integrating a measure of SNR as a function of gain, noise figure and decoupling into the Smith Chart.

\section{RESUlts}

The implemented decoupling circuit is found in Fig. 6 which is designed for $300 \mathrm{MHz}$. The coil is $5 \mathrm{~cm}$ in diameter using copper wire with a $3.2 \mathrm{~mm}$ thickness, mounted on plexiglass as seen in Fig. 7.

A number of practical aspects complicate the implementation of the matching circuit. Active decoupling (protection) is required to not impair the homogeneity of the transmitting coil and to prevent damaging the preamplifier. It works, in this case, by adding a parallel resonant circuit consisting of $C_{4}$ and $L_{1}$ in series with the coil to block the current at the frequency of interest. The inductor is switched on by the PIN diode $D_{1}$ which is activated by a DC signal fed through $L_{3}$. $C_{5}$ filters the DC path to avoid noise injection. 


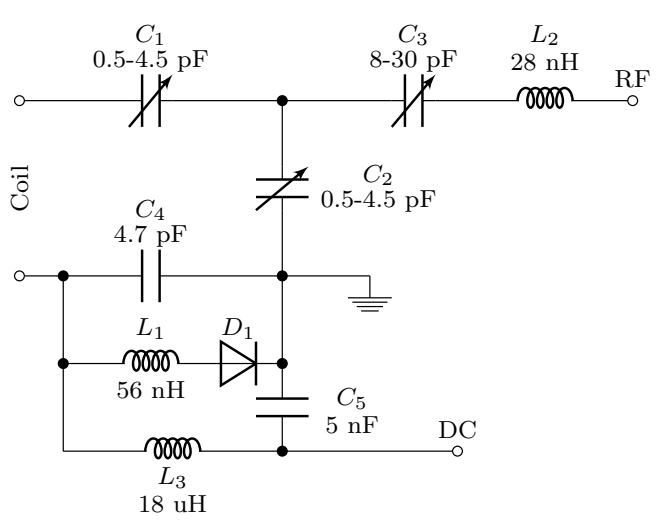

Figure 6. Final decoupling circuit including tunable components and active decoupling.

Capacitors $C_{1}, C_{2}$ and $C_{3}$ enable fine tuning of both the match and the decoupling. An issue with the series decoupling network is that matching and decoupling are correlated. This causes the procedure for tuning of the circuit to become tedious. First, the impedance is tuned using a network analyzer connected at the cable trap, see Fig. 7. Then, the decoupling is measured using the principle of reciprocity detailed in [11]-[13] with the preamplifier connected. This procedure is repeated until both matching and decoupling has been achieved. Further, a cable trap has been devised in order to block common mode current from flowing on the shield of the coaxial wire. The cable trap adds an inductance of approximately $10 \mathrm{nH}$ between the decoupling circuit and the preamplifier.

The matching impedance was chosen at $50 \Omega$ and the decoupling compared relative to this. The matching impedance for the comparison is $60.5+j 139 \Omega$ showing a relative increase in decoupling by $2.3 \mathrm{~dB}$. Theoretically the decoupling should be $3.3 \mathrm{~dB}$. The theoretical decoupling is not observed because of two aspects, 1) measurement uncertainty because the measurement is at the edge of the equipments range, 2) losses and tolerances of the components are not taken into account in the model. The optimal impedance for decoupling is not reached because the matching and decoupling is tuned using the same components. Hence, when the decoupling is optimal, the matching is skewed and vice-versa. This highlights the practical difficulty in tuning the series decoupling network to the optimum state.

\section{Conclusions}

Formulas for the series decoupling network for arbitrary matching and preamplifier impedances have been derived. This is used to show that matching to a different impedance than $50 \Omega$ achieves additional decoupling of surface coil elements while preserving the noise figure of the preamplifier. The work shows that for a given preamplifier, which was not designed for the purpose of decoupling, the optimal matching in terms of decoupling is indeed different from $50 \Omega$. Simulations show a potential of $5.6 \mathrm{~dB}$ additional decoupling for the

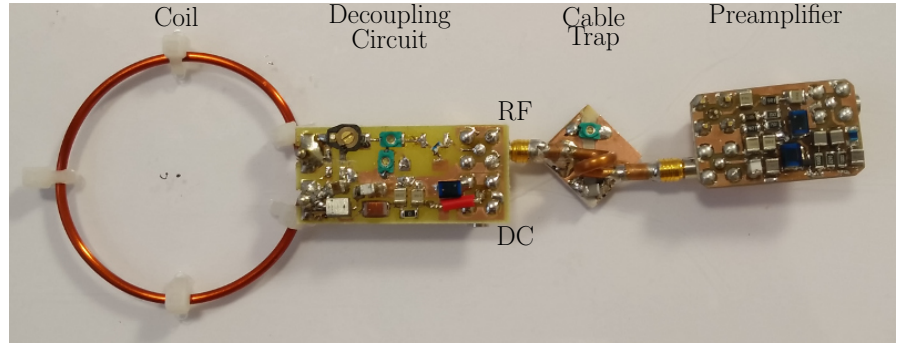

Figure 7. Setup with the coil, decoupling circuit, cable trap and preamplifier.

described setup. Measurements confirm the simulations by showing a $2.3 \mathrm{~dB}$ increase in decoupling. However, this was not at the optimal matching impedance. The optimal matching impedance was not achieved due to the practical difficulties in tuning the series decoupling circuit.

Having showed a proof-of-concept, the fundamental tradeoff between noise, decoupling and gain of preamplifiers for MRI can now be examined in detail analytically. We are now looking towards better integration and co-design of preamplifiers and decoupling circuits.

\section{ACKNOWLEDGEMENTS}

The authors would like to thank the Danish National Research Foundation (grant DNRF124) for partial support of the activities.

\section{REFERENCES}

[1] D. Erdogmus, E. G. Larsson, R. Yan, J. C. Principe, and R. Fitzsimmons, "Measuring the signal-to-noise ratio in magnetic resonance imaging: a caveat," Signal Processing, vol. 84, pp. 1035-1040, 2004.

[2] C.-N. Chen and D. I. Hoult, Biomedical Magnetic Resonance Technology, 1st ed. Institute of Physics Publishing, 1989.

[3] D. G. Nishimura, Principles of Magnetic Resonance Imaging, 1st ed. lulu.com, 2010.

[4] P. B. Roemer, W. A. Edelstein, and C. E. Hayes, "The NMR Phased Array," Soc. Magn. Reson. Med., vol. 225, pp. 192-225, 1990.

[5] K. P. Pruessmann, M. Weiger, M. B. Scheidegger, and P. Boesiger, "SENSE sensitivity encoding for fast MRI.pdf," Soc. Magn. Reson. Med., vol. 42, no. 5, pp. 952-962, 1999.

[6] J. B. Andersen and H. H. Rasmussen, "Decoupling and Descattering Networks for Antennas,' IEEE Trans. Antennas Propag., vol. 24, no. 6, pp. 841-846, 1976.

[7] J. Weber, C. Volmer, K. Blau, R. Stephan, and M. A. Hein, "Miniaturized antenna arrays using decoupling networks with realistic elements," IEEE Trans. Microw. Theory Tech., vol. 54, no. 6, pp. 2733-2740, 2006.

[8] J. C. Coetzee and Y. Yu, "Design of decoupling networks for circulant symmetric antenna arrays," IEEE Antennas Wirel. Propag. Lett., vol. 8, no. 2, pp. 291-294, 2009.

[9] A. Reykowski, S. M. Wright, and J. R. Porter, "Design of Matching Networks for Low Noise Preamplifiers," Magn. Reson. Med., vol. 33, no. 6 , pp. $848-852,1995$.

[10] D. Johansen, J. Sánchez-Heredia, V. Zhurbenko, and J. ArdenkjærLarsen, "Practical Aspects of Preamplifier Designs for 13C Imaging," in ISMRM 25th, 2017.

[11] D. Hoult and R. Richards, "The signal-to-noise ratio of the nuclear magnetic resonance experiment," J. Magn. Reson., vol. 24, no. 1, pp. $71-85,1976$

[12] D. I. Hoult and P. C. Lauterbur, "The sensitivity of the zeugmatographic experiment involving human samples," J. Magn. Reson., vol. 34, no. 2, pp. 425-433, 1979.

[13] D. I. Hoult, "The principle of reciprocity in signal strength calculations-A mathematical guide," Concepts Magn. Reson., vol. 12, no. 4 pp. 173-187, 2000. 